\title{
Cerebral Hemorrhage Due to Posterior Reversible Encephalopathy Syndrome Associated With Autonomic Dysreflexia in a Spinal Cord Injury Patient
} -Case Report-

\author{
Tae YAMASHita, ${ }^{1}$ Hisaya HiRAMATSU, ${ }^{1}$ Naoto SAKAI, ${ }^{1}$ and Hiroki NAMBA ${ }^{1}$
}

${ }^{1}$ Department of Neurosurgery, Hamamatsu University School of Medicine, Hamamatsu, Shizuoka

\begin{abstract}
A 37-year-old man with C4-5 spinal cord injury (SCI) presented with abnormally high blood pressure after vesicocutaneous catheter exchange and was treated with antihypertensive agents. Two weeks later, he developed headache and visual disturbance, and presented with fluctuating blood pressure. Multiple subcortical hemorrhages in the left occipital and right frontal lobes occurred on the next day, and he died of increased intracranial pressure 3 weeks later. Based on the symptoms and computed tomography findings, the retrospective diagnosis was posterior reversible encephalopathy syndrome (PRES) due to autonomic dysreflexia (AD). AD occurs frequently in patients with quadriplegia and high paraplegia by distention of the bladder and bowel. PRES secondary to AD is very rare, but we must always be aware of this life-threatening complication in SCI patients.
\end{abstract}

Key words: cerebral hemorrhage, posterior reversible encephalopathy syndrome, autonomic dysreflexia, spinal cord injury, vesicocutaneous catheter

\section{Introduction}

Cervical and high thoracic spinal cord injury (SCI) may be associated with significant dysfunction of the sympathetic nervous system. Autonomic dysreflexia (AD) is one of the life-threatening phenomena following this dysfunction. ${ }^{21)}$ AD manifests as complex symptoms characterized by sudden hypertension, with headache, bradycardia, sweating, and facial flushing, in response to stimulation of the organs below the levels of the SCI. ${ }^{2}$ AD mainly occurs in the chronic stage of SCI in around $50-80 \%$ of patients with quadriplegia and high paraplegia. ${ }^{18)}$ The main triggering factors are bladder over-distention or urinary tract infection and bowel distention. ${ }^{10,17)}$ Furthermore, iatrogenic triggering factors, such as cystoscopy, cystometry, or electrical stimulation of muscles, are also known. ${ }^{15}$ ) Intracranial hemorrhage is a rare complication of $\mathrm{AD}^{6,9,19,22-24)}$ with only a few cases of fatal massive hemorrhage secondary to AD. ${ }^{6,9,24)}$ We treated a patient with quadriparesis after SCI who developed multiple intracranial hemorrhages, which seemed to be related to posterior reversible encephalopathy syndrome (PRES) secondary to AD.

\section{Case Report}

A 37-year-old man suffered C4-5 American Spinal Injury

Received November 15, 2011;

Accepted December 9, 2011
Association grade B quadriplegia caused by a traffic accident at the age of 27 years. After SCI, he had neurogenic bladder and underwent cystostomy. The vesicocutaneous catheter was changed every 4 weeks at the hospital. He also suffered from constipation and spasticity in his extremities but he had no history of hypertension. His urethra was accidentally injured with the balloon of a new vesicocutaneous catheter during the periodic change. Another urethral catheter was additionally inserted to exert pressure on the wound. His systolic blood pressure elevated to $188 \mathrm{mmHg}$ at this time, but then declined to $140 \mathrm{mmHg}$ with nicardipine administration. He went back home without additional problems. However, his blood pressure fluctuated up and down and he often complained of headache. The urethral catheter was removed one week later and his headache decreased.

He began to suffer from headache and visual disturbance after one more week. Head computed tomography (CT) was performed, and he went home. He could eat and talk on the same day. Next evening, he began to vomit many times and his consciousness level deteriorated rapidly, so he was transferred to our hospital by ambulance (Glasgow Coma Scale E3V1M4). His blood pressure fluctuated widely with sinus bradycardia. Cramp or seizure was observed in his left upper limb. CT revealed multiple subcortical hemorrhages in the left occipital and right frontal lobes with perifocal edema (Fig. 1 upper row). Subsequent fluid-attenuated inversion recovery magnetic resonance (MR) imaging showed high intensity areas in 

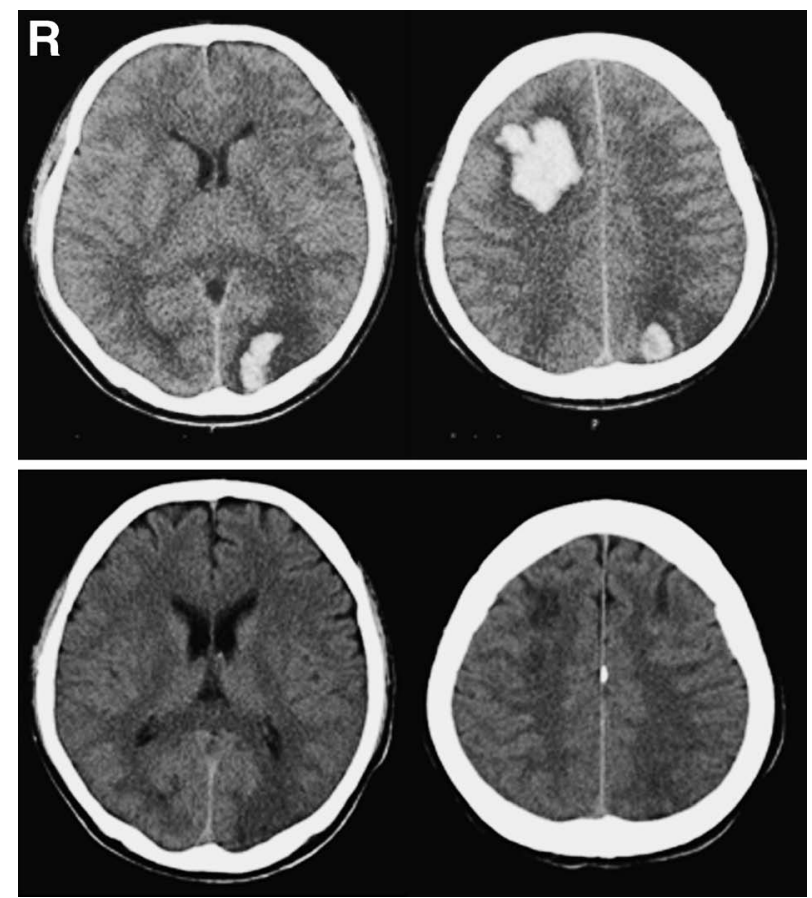

Fig. 1 Upper row: Computed tomography scans taken when the consciousness of the patient deteriorated showing massive subcortical hemorrhages and perifocal edema. Lower row: Computed tomography scans taken 2 days previously showing slightly low density areas in the bilateral occipital and frontal lobes.
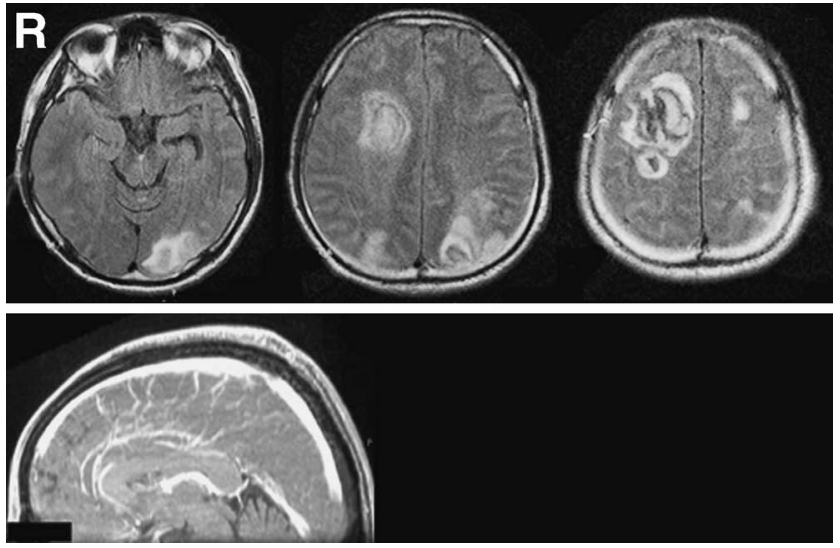

Fig. 2 Upper row: Fluid-attenuated inversion recovery magnetic resonance images showing asymmetrical bilateral occipital and frontal high density areas. Lower row: Enhanced magnetic resonance venogram showing patency of the superior sagittal sinus and cortical veins.

the asymmetrical bilateral occipital and frontal lobes (Fig. 2 upper row). MR angiography showed no vascular anomalies, such as moyamoya disease or arteriovenous malformation, and no occlusion or severe stenosis of the proximal arteries. Enhanced MR venography demonstrated patency of the superior sagittal sinus and cortical veins

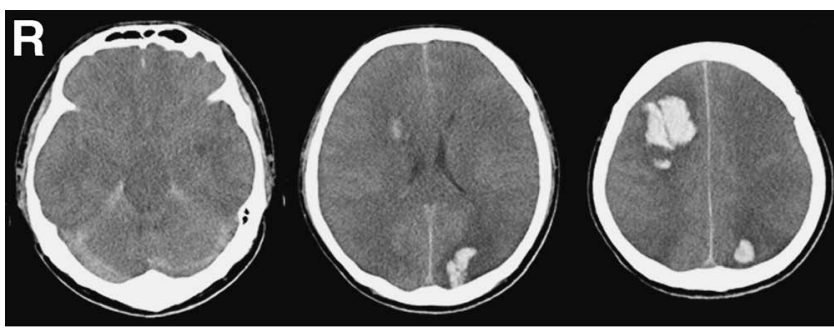

Fig. 3 Computed tomography scans taken when the patient became comatose with dilation of the bilateral pupils showing massive brain edema with transtentorial herniation.

(Fig. 2 lower row). Unfortunately, we could not evaluate the vasoconstriction of intracranial arteries because of poor MR angiography imaging due to motion artifacts caused by the patient. Inspection of the initial CT taken 2 days previously found low density areas in the bilateral occipital and frontal lobes (Fig. 1 lower row).

Laboratory investigations showed no evidence of coagulopathy, bleeding tendency, or thrombokinesis. MR venography findings and no elevation of D-dimer level excluded cerebral venous sinus thrombosis. We could not find any other risk factors to explain the multiple cerebral hemorrhages. Therefore, we diagnosed cerebral hemorrhage secondary to $\mathrm{AD}$. He was conservatively treated with anticonvulsants and glycerol. After hospitalization, his blood pressure was around $120 / 70 \mathrm{mmHg}$ without antihypertensive agents. No other sympathomimetic symptoms such as sweating, facial flushing, or nasal stuffiness were noted. After several hours, his bilateral pupils dilated and he became comatose with periodic respiration. CT showed progressive brain edema and transtentorial herniation (Fig. 3). He was immediately intubated and mechanically ventilated, but his neurological status did not recover and he died 3 weeks after the intracranial hemorrhage. His family refused to permit autopsy.

\section{Discussion}

Hypertension due to AD is the cause of much morbidity and mortality, so adequate blood pressure reduction is necessary. ${ }^{2)}$ This disorder should be promptly recognized and treated because antihypertensive therapy, in addition to removal of the trigger factors, can usually control the hypertension due to AD. ${ }^{14)}$ However, management of the blood pressure of SCI patients is sometimes difficult, as in the present case, so prevention of $\mathrm{AD}$ is more important. The medical team, as well as the SCI patients themselves, should be aware of this disorder, and appropriate bladder care, bowel training, and skin care should be provided. If paradoxical hypertension is noticed in SCI patients, blood pressure should be adequately controlled and the trigger factors should be immediately identified and resolved. Careful blood pressure control is necessary for prevention of further complications, because the blood pressure of SCI patients easily fluctuates. In particular, a patient who has experienced $\mathrm{AD}$ and become free from the trigger might have another episode of AD in around 1-2 weeks be- 
cause of an easily excitable autonomic nervous system. ${ }^{22}$ ) In fact, a life-threatening episode of AD occurred 2 weeks after the initial minor episode in the present patient. We definitely need to recognize the risk of AD recurrence.

In the present case, we retrospectively found bilateral occipital and frontal low density areas on the initial CT suggesting PRES, when the patient displayed symptoms such as abrupt hypertension, headache, and visual disturbance. PRES was first described in 1996 ${ }^{13)}$ and recently has been widely recognized as clinical manifestations such as headache, seizure, visual change, and altered mentation. The most common causes of PRES are hypertensive encephalopathy, eclampsia, chemotherapeutic agents, and antirejection therapy. PRES is usually reversible, but sometimes results in irreversible neurological deficits. The mechanism of PRES remains controversial. Two theories have been generally proposed: severe hypertension leads to failed auto-regulation and hyperperfusion with endothelial injury and vasogenic edema; or vasoconstriction and hypoperfusion leads to brain ischemia and subsequent vasogenic edema. ${ }^{1)}$ The typical neuroimaging findings of PRES are predominantly posterior subcortical vasogenic edema without infarction, but the anterior circulation territory is sometimes affected.11) The prevalence of intracranial hemorrhage in PRES was reported as $5-19 \% .{ }^{12,16,20)}$ Intracranial hemorrhage is often multifocal and within the regions of parenchymal edema. Endothelial injury and breakdown of the blood-brain barrier may be important as possible factors contributing to hemorrhage. ${ }^{20}$ The cause of the severe brain edema in the present case was mostly unknown, because no episodes of high and/or low blood pressure, or hypoxia were noted after hospitalization. Dysfunction in the autonomic system of the cerebral vasculatures might have been involved.

PRES seems to have co-morbidity with reversible cerebral vasoconstriction syndrome (RCVS). ${ }^{5}$ ) RCVS is a group of disorders characterized by acute onset, severe headaches, and reversible vasoconstriction of the cerebral arteries with or without neurological defects. ${ }^{3)}$ The relationship between RCVS and PRES remains unclear, but both syndromes are thought to involve disturbances in cerebral arterial tone as part of the pathophysiological basis. ${ }^{3)}$ One third of all cases of RCVS involved intracranial hemorrhage in prospective analysis. ${ }^{7)}$ In the present case, constriction of the cerebral arteries could not be correctly evaluated because of the motion artifacts on MR angiography, but RCVS caused by abnormal sympathetic activities in AD might have occurred.

PRES or RCVS secondary to AD is very rare, with only two case reports. ${ }^{4,8}$ One patient developed a large cerebral infarction, ${ }^{8)}$ but the other recovered without sequelae. ${ }^{4}$ Prompt recognition of these syndromes is important to prevent further morbidity and mortality in SCI patients. In the present case, PRES should have been suspected from the initial CT when the patient complained of headache and visual disturbance 2 days before the cerebral hemorrhage. If a patient presents with symptoms indicative of PRES, MR imaging and MR angiography, in addition to $\mathrm{CT}$, are recommended.

In conclusion, we must always be aware that patients with SCI, especially in the high spinal levels, could easily develop AD caused by the stimulation of the lower parts of the body during life. If patients have symptoms such as paradoxical hypertension, headache, or facial flushing, prompt removal of the triggers and careful control of blood pressure are very important. Prevention of AD occurrence is more important, because intensive care sometimes fails to save patients with life-threatening complications such as intracranial hemorrhage.

\section{References}

1) Bartynski W: Posterior reversible encephalopathy syndrome, part 2: controversies surrounding pathophysiology of vasogenic edema. AJNR Am J Neuroradiol 29: 1043-1049, 2008

2) Braddom R, Rocco J: Autonomic dysreflexia. A survey of current treatment. Am J Phys Med Rehabil 70: 234-241, 1991

3) Calabrese L, Dodick D, Schwedt T, Singhal A: Narrative review: reversible cerebral vasoconstriction syndromes. Ann Intern Med 146: 34-44, 2007

4) Chaves C, Lee G: Reversible posterior leukoencephalopathy in a patient with autonomic dysreflexia: a case report. Spinal Cord 46: 760-761, 2008

5) Chen S, Fuh J, Wang S: Reversible cerebral vasoconstriction syndrome: an under-recognized clinical emergency. Ther Adv Neurol Disord 3: 161-171, 2010

6) Dolinak D, Balraj E: Autonomic dysreflexia and sudden death in people with traumatic spinal cord injury. Am J Forensic Med Pathol 28: 95-98, 2007

7) Ducros A, Fiedler U, Porcher R, Boukobza M, Stapf C, Bousser M: Hemorrhagic manifestations of reversible cerebral vasoconstriction syndrome: frequency, features, and risk factors. Stroke 41: 2505-2511, 2010

8) Edvardsson B, Persson S: Reversible cerebral vasoconstriction syndrome associated with autonomic dysreflexia. J Headache Pain 11: 277-280, 2010

9) Eltorai I, Kim R, Vulpe M, Kasravi H, Ho W: Fatal cerebral hemorrhage due to autonomic dysreflexia in a tetraplegic patient: case report and review. Paraplegia 30: 355-360, 1992

10) Erickson R: Autonomic hyperreflexia: pathophysiology and medical management. Arch Phys Med Rehabil 61: 431-440, 1980

11) Gocmen R, Ozgen B, Oguz K: Widening the spectrum of PRES: series from a tertiary care center. Eur J Radiol 62: 454-459, 2007

12) Hefzy H, Bartynski W, Boardman J, Lacomis D: Hemorrhage in posterior reversible encephalopathy syndrome: imaging and clinical features. AJNR Am J Neuroradiol 30: 1371-1379, 2009

13) Hinchey J, Chaves $C$, Appignani B, Breen J, Pao L, Wang A, Pessin M, Lamy C, Mas J, Caplan L: A reversible posterior leukoencephalopathy syndrome. N Engl J Med 334: 494-500, 1996

14) Karlsson A: Autonomic dysreflexia. Spinal Cord 37: 383-391, 1999

15) Krassioukov AV, Karlsson AK, Wecht JM, Wuermser LA, Mathias CJ, Marino RJ; Joint Committee of American Spinal Injury Association and International Spinal Cord Society: Assessment of autonomic dysfunction following spinal cord injury: rationale for additions to International Standards for Neurological Assessment. J Rehabil Res Dev 44: 103-112, 2007

16) Lee VH, Wijdicks EF, Manno EM, Rabinstein AA: Clinical 
spectrum of reversible posterior leukoencephalopathy syndrome. Arch Neurol 65: 205-210, 2008

17) Lindan R, Joiner E, Freehafer A, Hazel C: Incidence and clinical features of autonomic dysreflexia in patients with spinal cord injury. Paraplegia 18: 285-292, 1980

18) McKinley W, Jackson A, Cardenas D, DeVivo M: Long-term medical complications after traumatic spinal cord injury: a regional model systems analysis. Arch Phys Med Rehabil 80: 1402-1410, 1999

19) Pan $\mathrm{S}$, Wang $\mathrm{Y}$, Lin $\mathrm{H}$, Chang $\mathrm{C}$, $\mathrm{Wu} \mathrm{T}$, Hsieh $\mathrm{E}$ : Intracerebral hemorrhage secondary to autonomic dysreflexia in a young person with incomplete C8 tetraplegia: A case report. Arch Phys Med Rehabil 86: 591-593, 2005

20) Sharma A, Whitesell R, Moran K: Imaging pattern of intracranial hemorrhage in the setting of posterior reversible encephalopathy syndrome. Neuroradiology 52: 855-863, 2009

21) Teasell R, Arnold J, Krassioukov A, Delaney G: Cardiovascular consequences of loss of supraspinal control of the sympathetic nervous system after spinal cord injury. Arch Phys
Med Rehabil 81: 506-516, 2000

22) Vallès M, Benito J, Portell E, Vidal J: Cerebral hemorrhage due to autonomic dysreflexia in a spinal cord injury patient. Spinal Cord 43: 738-740, 2005

23) Yokomizo Y, Goubara A, Tanaka K, Yokoyama O: [A case of cerebellar hemorrhage secondary to autonomic dysreflexia (AD) in a patient with cervical spinal cord injury]. Hinyokika Kiyo 56: 659-661, 2010 (Japanese)

24) Yoo K, Jeong C, Kim W, Lee H, Kim S, Jeong S, Lee J, Lee J: Fatal cerebral hemorrhage associated with autonomic hyperreflexia during surgery in the prone position in a quadriplegic patient: a case report. Minerva Anestesiol 76: 554-558, 2010

Address reprint requests to: Hiroki Namba, MD, Department of Neurosurgery, Hamamatsu University School of Medicine, 1-20-1 Handayama, Higashi-ku, Hamamatsu, Shizuoka 431-3192, Japan.

e-mail: hnamba@hama-med.ac.jp 